\title{
Populäre Sachprosa und naturwissenschaftliche Sprache
}

\author{
Dargestellt am Beispiel eines Postversandbuchs vom Verlag Das \\ Beste und eines erzählerischen Sachbuchs von Hoimar von \\ Ditfurth [1980]
}

Im 18. Jahrhundert ging die Wissenschaftssprache in Deutschland vom gelehrten Latein zur Volkssprache über. Dieser Übergang wurde am Ende des 17. Jahrhunderts von Thomasius und Leibniz ${ }^{1}$ unter dem Leitgedanken einer allgemeinen nationalen Aufklärung gefordert. Er brachte unter anderem die bewunderte Fachprosa des 19. Jahrhunderts hervor.

Ebenfalls im 18. Jahrhundert, als das Interesse der Gebildeten an den Wissenschaften, insbesondere auch den Naturwissenschaften, zunahm, begann ein zweiter Übersetzungsvorgang, der von dem gleichen aufklärerischen Impuls getragen war: die Übertragung des Wissenswerten aus den deutschen, sich von der Gemeinsprache entfernenden Fachsprachen in eine auf ein allgemeines Lesepublikum berechnete populäre Prosa. Dieser zweite Übersetzungsvorgang (vgl. Wetzels 1971) ist im 19. und 20. Jahrhundert um so wichtiger geworden, als die Kluft zwischen Fachsprachen und Gemeinsprache sich ständig erweitert hat.

Der Vorzug einer solchen, der Differenzierung unsrer Sprache entgegenwirkenden Anstrengung liegt darin, dass sie fachsprachlich vorformulierte Inhalte von der Gemeinsprache her neu durchdenkt und sie allgemeiner zugänglich macht, dass sie die von der Aufklärung geforderte Teilhabe aller an dem von den Wissenschaften Erarbeiteten, den Austausch, die Zirkulation der geistigen Güter ermöglicht. Eine differenzierte Ausarbeitung der Gemeinsprache und ihre Bereicherung um neue Begriffe ist die Folge.

Diese Leistung wird heute getragen von den Medien - Zeitung, Rundfunk, Fernsehen - und ihren nicht allzu dicht gesäten Wissenschaftsjournalisten, dem Unterricht und den Verfassern von Schulbüchern, den Verlagen von Sachbüchern und populären Zeitschriften und ihren Autoren, und schließlich von den gemeinverständlich schreibenden Wissenschaftlern. Die letzte Gruppe scheint die kleinste $\mathrm{zu}$ sein, teils weil ein populär schreibender Gelehrter bei uns eher einen

1 Vgl. Thomasius 1970: 21-25 (Discours. Welcher Gestalt man denen Frantzosen in gemeinem Leben und Wandel nachahmen solle); Thomasius 1692: 13 u. ö. (Einleitung zur Vernunftlehre, Vorrede, §4); Leibniz 1916a: 13ff. (Ermahnung an die Deutschen); Leibniz 1916a: $26 \mathrm{ff}$. (Von deutscher Sprachpflege, §§ 8ff.).

Ә Open Access. (C) Uwe Pörksen, publiziert von De Gruyter. (c) BY-NC-ND Dieses Werk ist lizenziert unter der Creative Commons Attribution-NonCommercial-NoDerivatives 4.0 Lizenz. 
Namen zu verlieren hat, teils weil $\mathrm{u}$. U. die Vorgaben des Verlags ihn bei der Niederschrift eines Sachbuchs allzu sehr einengen.

Die Sprachwissenschaft ist an diesem Übersetzungsvorgang überhaupt nicht beteiligt. Das ist, gelinde gesagt, auffällig. - Es ist auch sehr die Frage, ob ein interdisziplinäres deutsches Wörterbuch (vgl. Henne 1978), von Harald Weinrich 1975 vorgeschlagen und seither diskutiert, angesichts der Geschwindigkeit, mit der sich gegenwärtig das lexikonwürdige Wissen erweitert - es verdoppelt sich in einem Zyklus von vier bis fünf Jahren - und der Langsamkeit, mit der Philologen ein Wörterbuch zu erstellen pflegen, die Chance hätte, $\mathrm{zu}$ einem Zeitpunkt zu erscheinen, an dem es nicht schon veraltet wäre.

Bevor von den sprachlichen Mitteln die Rede ist, die man beim Übergang wissenschaftlicher Texte in populäre Sachprosa beobachten kann, möchte ich einige Bemerkungen zur Klassifikation der Sachliteratur und zu den Rahmenbedingungen ihrer Herstellung machen. Eine Klassifizierung der auf dem Markt befindlichen Buchtypen ist nicht einfach, und die Rahmenbedingungen ihrer Herstellung sind von denen der institutionell und finanziell abgesicherten Ausnahmebedingung wissenschaftlicher Veröffentlichungen grundverschieden und so geartet, dass sie sich auf Sprache und Gestaltung der Sachbücher tiefgreifend auswirken müssen.

\section{Zur Klassifikation der Sachbücher und den Rahmenbedingungen ihrer Produktion}

Das Sachbuch - wenn man es abgrenzt gegen die Belletristik - macht $80 \%$ der heute produzierten Literatur aus; es gibt kaum einen größeren Verlag, der nicht auch Sachliteratur herstellt. Man spricht von ,Ratgebern‘ (für Garten, Basteln, Gesundheit, Autoreparatur), vom ,Bildsachbuch“ (Natur und Kunst sind beliebte Gebiete), vom ,erzählerischen Sachbuch` (das bahnbrechende Werk Götter, Gräber und Gelehrte hat den bezeichnenden Untertitel Ein Roman der Archäologie), vom ,Enzyklopädischen Sachbuch` (einem systematisch angelegten Werk mit hohem wissenschaftlichem Anspruch), vom ,kleinen Fachlexikon‘.

Als Anhalt für die Klassifikation eignen sich vielleicht folgende Gesichtspunkte: ${ }^{2}$

2 Ich folge hier einer Anregung des Leiters der Informationsabteilung des Herder Verlags, Herrn Dr. Udo Becker. 
1. das Verhältnis von Information und Redundanz. Das erzählerische Sachbuch hat eine sehr viel höhere Redundanz als die Enzyklopädie, diese wiederum eine höhere als das Lexikon;

2. die übersichtliche Anordnung der Information, Zugriffswahrscheinlichkeit und Zugriffszeit. Sie ist beim Lexikon sehr ausgeprägt, im erzählerischen Sachbuch ist die Information verstreut;

3. der Anspruch, das Niveau der sachlichen und sprachlichen Voraussetzungen und die Kompliziertheit der Darstellung. Von einem gemeinsprachlichen reich bebilderten Sachbuch wie Tiergiganten bis zu dem enzyklopädischen Sachbuch Erforschter Weltraum gibt es auch hier ein breites Spektrum; ${ }^{3}$

4. der Anteil der optischen Vermittlung von Information und auch die Art der Verbildlichung. Auch hier lässt sich die Unterscheidung von Information und Redundanz anwenden. Zeichnungen, Diagramme sind informationshaltig, die Farbfotographie oft nur ein Stimmungsträger, und zwischen beiden gibt es Zwischenstufen und Kombinationen. ${ }^{4}$

Ein Verlag wie Herder (Freiburg) hat als Zielpublikum ein differenziertes Spektrum und stellt eine Reihe entsprechend verschiedener Buchtypen her; ein Verlag wie „Das Beste“ in Stuttgart wendet sich an ein breites einheitliches Publikum und entsprechend einheitlich sind die Sachbücher des Verlags, z.B. in der Art, dass der Bildanteil ein Drittel ausmacht. Ein normales Sachbuch hat hier eine Auflage von 300000 bis 400000, ein erfolgreiches erreicht 800000 bis eine Million. ${ }^{5}$

Sachbücher werden vielfach auf der Grundlage internationaler Kooperation hergestellt - Bildmaterial wird ausgetauscht, Werke werden als Ganzes adaptiert, um Lokalkolorit und Vorurteilsstrukturen des Herkunftslandes beschnitten und um die des Ankunftslandes bereichert, oder sie werden von vornherein auf ihre internationale Verwertbarkeit, u. U. mehrsprachig konzipiert.

Das klassische Beispiel, ein Autor schreibt ein Buch, trifft nicht mehr zu. In 90 bis 100 \% der Fälle konzipiert der Verlag das Buch, entwickeln Redakteur oder Lektor und Produktionsleiter, Marktforscher und Vertrieb in rivalisierender Zu-

3 Auch der Aspekt des Schwierigkeitsgrades ist nicht unabhängig von Informationsdichte bzw. Redundanz zu sehen. Denn in der Regel gilt (oder sollte doch gelten): je allgemeiner und redundanter ein Text um so leichter zugänglich, je spezieller und informationsdichter, um so schwieriger.

4 Sehr lehrreich in diesem Zusammenhang ist Bertin 1974.

5 Einem aufschlussreichen Artikel von Mörmann 1976 entnehme ich einige Daten. Herrn Dr. Hans Dieter Hänel, Redakteur beim Verlag Das Beste in Stuttgart, danke ich für zahlreiche Hinweise und bereitwillige Auskunft. 
sammenarbeit das intellektuelle und technische Modell. Ehe Autoren engagiert werden bzw. unabhängig von ihnen, liegt das meiste fest: vom Titel über die Kapiteleinteilung und den Bildanteil bis zur Seitenzahl der Kapitel. Die Herstellung ist extrem arbeitsteilig. Die Entwicklung eines Buches dauert drei bis fünf Jahre, seine durchschnittliche Lebensdauer beträgt ebenfalls drei Jahre.

Buchidee und -ausführung sind $\mathrm{zu}$ einem hohen Grad marktbestimmt. Das Auffinden der Marktnische und die Analyse des Zielpublikums stehen meist am Anfang. - Bei Verlagen, deren Vertriebsform der Postversand ist, steht der Test im Zentrum der ganzen Herstellung; das Endprodukt wird in einer Serie von Tests herausgefiltert. $^{6}$

Nachdem der Autor im Entstehungsprozess nach hinten rückt, wer verfasst die Sachliteratur? In diesem Punkt waren die Auskünfte unserer Gewährsleute etwas unbestimmt. Die Hauptarbeit wird offenbar von Mitgliedern des Verlags, Lektoren und Redakteuren, geleistet oder von Fachleuten am Feierabend und nach der Pensionierung.

Der Verlag nennt gewisse Rahmenbedingungen für die Schreibweise, die dann am Manuskript überprüft werden. Die Vorgaben sind anscheinend oft nur vage formuliert: der Autor soll ,allgemeinverständlich für interessierte Laien“ schreiben, ein Fachbuch soll ,rund“, der Stil ,griffig“ sein. - Der Verlag „Das Beste“ verfügt offenbar über das am meisten ausgearbeitete System von Schreibkonventionen und -regeln.

Das Manuskript wird vom Verlag nachgeprüft und überarbeitet, eventuell ganz umgearbeitet. Die Normen, denen ein Redakteur oder Lektor folgt, sind in der Regel offenbar kein Kodex formulierter Regeln - sie werden beim Eintritt in den Verlag, der u. U. seine besonderen Schreibkonventionen und ,Strickmuster hat, am praktischen Einzelfall erlernt und so unbewusst gehandhabt wie die Regeln der Grammatik auch.

Der erklärte Zweck dieser Literatur ist die Vermittlung von Sachwissen, von Information; die Hauptintention bei ihrer Herstellung ist in der Regel die Erwirtschaftung von Profit, der Verkauf. Damit verschiebt sich das Interesse in doppel-

\footnotetext{
6 Das Ergebnis beschreibt Werner Waldmann (zit. nach Mörmann 1976: 202): „Das Programm der Mail-Order-Firmen umfaßt nur solche Titel, die entweder wertneutral Fakten und Hilfestellung bieten oder die Sonnenseiten dieser Welt auf Hochglanzseiten konsumierbar machen.“ „Die Verlage haben kein Interesse daran (und mit diesem System auch kaum eine Möglichkeit) die Käufer für neue Themen und Probleme zu gewinnen, sozusagen didaktisch zu wirken. Die Werbung hat da ihre Grenzen, wo sie nicht aus bestehenden Bedürfnissen, auch unbewußten, ein konkretes Verlangen modulieren kann.“
} 
ter Hinsicht von der Sachorientierung auf die Publikumsorientierung. Die Übertragung wissenschaftlicher Prosa in ein populärwissenschaftliches Sachbuch besteht nur z. T. in einem ,Übersetzen“; in der Hauptsache ist es ein Vorgang der Umarbeitung aus einem überwiegend der Sacherschließung dienenden Darstellungstyp in einen publikumsorientierten.

Gibt es übereinstimmende sprachliche und darstellungstechnische Mittel bei diesem Umsetzungsvorgang?

\section{Sprachliche und darstellungstechnische Mittel bei der Umsetzung wissenschaftlicher in populärwissenschaftliche Literatur}

\section{a) Bearbeitungsstufen eines Postversandbuchs (Die Naturwunder der Erde)}

Das Buch Die Naturwunder der Erde, das 1979 vom Verlag „Das Beste“ in Stuttgart herausgebracht wurde, ist ein großformatiges Bildlexikon, das auf 450 Seiten von der Aareschlucht bis zur Zugspitze - 377 Landschaftserscheinungen darstellt. Das Buch enthält sicher mehr als ein Drittel an bildlicher Darstellung, 354 Farbfotos, 80 Karten und fast ebenso viele kolorierte Zeichnungen. Die Lexikoneinträge sind annähernd gleich lang und nach nur leicht variiertem Muster gebaut. Sie beginnen mit einer Kennzeichnung der geographischen Lage, einem Bildsymbol, das andeutet, ob von einer Insel, einem Vulkan, einer Kliffküste usw. die Rede ist, einem ebenso umrisshaften Vorspann, und informieren dann über Lage und Aussehen, Entstehung, Entdeckung und Erforschung, geologische und geographische Aspekte des Naturwunders.

Als allgemeinste strukturgebende Momente des Buches erscheinen Unterhaltung und Belehrung, Information und Anreiz. Dem Anreiz und der Unterhaltung dienen in erster Linie die prächtigen, ein universelles Fernweh erzeugenden Fotos, die Auswahl der ,großartigsten' und ,berühmtesten' Naturerscheinungen unsrer Erde, das den Superlativ bevorzugende und dramatisierende Vokabular, die bildstarke und freundliche Sprache. Der Information dienen vor allem die Kartenübersichten am Anfang, die oft in Boxen eingerahmten kolorierten Zeichnungen und verbalen Erläuterungen, die terminologiereichen einzelnen Artikel, eine 14seitige ,Erklärung wichtiger Fachausdrücke` und das Register am Schluss.

Das Buch ist eine Adaptierung des 1977 von der französischen Tochterfirma des Reader's Digest herausgebrachten Buches Dictionnaire Illustré des Merveilles 
Naturelles du Monde. Die deutsche Ausgabe ist vollständig überarbeitet worden. In einem Seminar über Sachliteratur, das ich im Wintersemester 78/79 gehalten habe, stellte uns Herr Dr. Hans Dieter Hänel, Redakteur beim Verlag „Das Beste“ in Stuttgart, freundlicherweise einiges Werkstattmaterial zu dem Artikel „Etna [Ätna]“ zur Verfügung, und zwar: die erste Bearbeitung (a) der wörtlichen Übersetzung aus dem Französischen, die erste Korrektur (b) dieser Bearbeitung und die zweite (und letzte) Korrektur (c). Die drei Bearbeitungsstufen ergeben einen guten Einblick in einige Arbeitsgrundsätze des Verlags. Ich greife nur Beispiele heraus. In der übersetzten französischen Fassung heißt es im Vorspann über den „Etna“: „Jede seiner Narben, jeder seiner Wülste, seiner eingestürzten Krater und ganz oben jeder seiner zum Himmel offenen Schlünde kündet von den verschlungenen Wegen eines gleichzeitig fantastischen und tragischen Schicksals. “ Es folgt das Zitat eines antiken Schriftstellers: sich ein Bild von der Natur zu machen, sei „für den Geist eine göttliche Beschäftigung“.

Die erste Bearbeitung (a) setzt im Vorspann stattdessen: „Trotz der Gefährlichkeit dieses Feuerberges sind seine fruchtbaren Hänge dicht besiedelt und von gepflegten Ölbaumhainen gesäumt.“ Der Rest ist ersatzlos gestrichen.

Die Bildunterschrift beginnt im Französischen: „Ätna. Altar des Hephaistos, aus dem mit seltsamem Leuchten die Lava austritt [...]“; in der Bearbeitung: „Ätna. Lavabomben, Steine und Aschen werden als feuerrote Glutwolke aus dem Hauptkrater in die klare Winterluft geschleudert [...]“.

Das antikisierende Pathos des französischen Textes wird also beseitigt und ersetzt durch sachliche Information bzw. durch einen Idyllik und Explosivität verbindenden Text, der den vermuteten Sehnsuchts- und Vorurteilsstrukturen des deutschen Publikums besser entspricht.

In der ersten Korrektur finden sich vor allem Eingriffe in die Syntax. Ein informationskompakter Satz - „Im höheren Vulkangestein versickernde Niederschläge kommen hier in zahlreichen Quellen zum Vorschein und ermöglichen eine intensive Bewässerung der fruchtbaren Lavaböden und damit eine Bevölkerungsdichte bis zu 500 Einwohnern pro $\mathrm{km}^{2 *}$ - dieser Satz wird aufgelöst in drei Hauptsätze: „Niederschlagwasser, das in der Gipfelregion versickert, tritt hier wieder an die Oberfläche. Diese Quellen bilden die Grundlage einer intensiven Bewässerung [...]. Die Bevölkerungsdichte beträgt [...].“

Aber auch das umgekehrte Prinzip wird angewandt. Drei einfach gebaute Hauptsätze werden - bei etwas verkürzter Information - umgeformt in einen Hauptsatz mit Nebensatz; oder ein Nebensatz (,„...] Gipfelkegel, der einen dreifach ineinandergeschachtelten Krater besitzt.“) wird lesbar gemacht, indem er in ein Attribut umgeformt wird (,[...] Gipfelkegel mit seinem mehrfach verschachtelten Krater"). Hatte schon die erste Bearbeitung (a) einiges an geologischer Information und Terminologie aus der französischen Fassung gestrichen, so ersetzt die 
erste Korrektur (b) z.B. ,sich versteilen` durch ,steiler werden', ,Plateau' durch ,Hochfläche‘.

Die letzte Korrektur (c) enthält nur noch wenige Zusätze, insbesondere Adjektive, die verdeutlichen und dramatisieren. Statt „Auf dieser weiten Plattform“ heißt es jetzt „Auf dieser deutlich ausgeprägten, von weitem erkennbaren Plattform“, statt „der große Ausbruch von 1669“ - „der große, verheerende Ausbruch von 1669“, statt „Lavaströme, die sich pausenlos zu Tal wälzten“ - „Lavaströme, die sich pausenlos mit großer Geschwindigkeit zu Tal wälzten.“

Ein Satz, den man als allgemeine wissenschaftliche Legitimationsformel bezeichnen könnte, wird neu in den Artikel eingeführt: „Den Geologen ist es gelungen, fast alle [erg.: Eruptionen] anhand von genauen Untersuchungen im Gelände nachzuweisen.“

Die sprachlichen Beobachtungen an dem Artikel „Etna“ und zu weiteren Artikeln des Bildlexikons ließen sich vervollständigen. Das soll aber in dem weiteren Rahmen eines Vergleichs zwischen naturwissenschaftlicher Sprache und populärer Sachprosa erfolgen.

\section{b) Sachliteratur mit breitem Zielpublikum und naturwissenschaftliche Sprache}

Wir haben in dem erwähnten Seminar einige Textstücke verglichen, die den gleichen Inhalt einmal in wissenschaftlicher und einmal in populärer Form darstellen: einen Abschnitt über Molekularbiologie der Zelle aus einem Handbuch und ein entsprechendes Stück aus Hoimar von Ditfurths Im Anfang war der Wasserstoff, einen Fachtext über die Radiokarbonmethode und einen entsprechenden Abschnitt aus dem Großen Reader's Digest Jugendbuch, etwas über Vulkanismus aus einem wissenschaftlichen Werk und aus der Zeitschrift GEO, Textstücke über Tuberkulose aus einem medizinischen Fachbuch Differentialdiagnose innerer Krankheiten und aus einem Ratgeber Sprechstunde für Gesunde und Kranke, u.a.m. Ich nehme hier noch die 1979 vom Verlag Das Beste herausgebrachten Sachbücher Die Naturwunder der Erde und Meilensteine des 20. Jahrhunderts hinzu. Das Material ist heterogen, aber einige lohnende Gesichtspunkte bzw. Verschiebungstendenzen, die häufiger zu beobachten sind, seien angeführt.

Die Adjektive nehmen an Zahl eher zu, aber neben oder an die Stelle der informierenden, orientierenden und charakterisierenden Adjektive des wissenschaftlichen Textes treten mehr Stellung nehmende, urteilende, wertende: auf der einen Seite z. B. ,katalytisch', ,flüssig‘, ,speziell` - auf der anderen Seite ,erstaunlich‘, ,geheimnisvoll‘, ,weltumspannend‘. Das Adjektiv dient oft einfach der Hervorhebung, der Verstärkung - ähnlich wie der Superlativ. 
In den Artikeln von Die Naturwunder der Erde stehen besonders am Anfang Adjektive dieses nur unterstreichenden Typs, ,berühmt‘, ,außergewöhnlich‘, ,gigantisch‘, ,gewaltig‘, ,phantastisch', ,prächtig‘, ,mächtig‘, ,wunderbar‘, häufig im Superlativ. („Der Ätna liegt an der ionischen Küste Siziliens und gehört zu den meisterforschten, bestbekannten, aber auch kompliziertesten Vulkanen der Erde“). Im weiteren Text finden sich eher informierende Adjektive, aber auch bildkräftige - ,grasiges Wasser‘, ,zerfurchte Hänge‘, ,eisbewehrte Steilhänge` werden gewählt.

$\mathrm{Zu}$ den Verben: ein Merkmal wissenschaftlicher Texte sind die sogenannten ,blassen', desemantisierten Verben, die fast nur noch eine grammatische, satzkonstituierende Funktion haben - ,teilnehmen', ,aufweisen', ,bilden“ - die populäre Prosa tendiert eher zu Verben der Aktion, die anschaulich sind, die innerhalb des Satzes geschehens- und bildkonstituierende Wirkung haben ,sich bedienen“, ,entwickeln', ,sich sträuben', ,aufdecken‘, ,herausfinden“, ,zurückreichen'.

Öfter ist der Bildgehalt anthropomorphisierend: die Natur ,entwickelt‘, ,bedient sich‘, ein Berg ,bringts‘ auf 10000 Meter.

Um auch gleich von den Verbformen und von syntaktischen Besonderheiten zu sprechen: für Texte naturwissenschaftlichen Inhalts ist an sich das zeitlose Sachverhalte bezeichnende Präsens typisch, in der populären Darstellung finden wir dagegen immer wieder das Präteritum. Die Darstellung geht in Erzählung über.

Der Konjunktiv und überhaupt das Modalfeld der Sprache, durch das wir den Sicherheitsgrad einer Aussage einschränken oder sie nur als denkmöglich hinstellen, ist im für die Allgemeinheit geschriebenen Text anscheinend viel seltener; die Passivkonstruktionen und Zustandsverben (,ist gegeben', ,liegt bei', ,sind beteiligt'), die ein Geschehen als Vorgang oder als Zustand abbilden, wobei das veranlassende Subjekt u. U. ausgespart wird, sind im wissenschaftlichen Text häufiger. Im populären Text werden Sachzusammenhänge weniger statisch abgebildet als dynamisiert, dramatisiert;

Partizip I und Partizip II haben im informationshaltigen Text ihre Funktion, da sie satzverkürzend verwendet werden können - eine Form wie ,bei mehrmals wiederholten Ergüssen' würde in einem populären Text vermutlich aufgelöst in den Nebensatz ,wenn sich die Ergüsse mehrmals wiederholen';

satzverkürzend wirkt sich auch der Gebrauch von nominalisierten Verben aus und hier dürfte das Gleiche gelten wie eben. Die Substantivgruppe ,Zur Messung derart geringer Zufallsraten`z.B. würde in der Regel zurückgeführt auf ihr Äquivalent in einem entsprechenden Nebensatz;

der Gebrauch von Abstrakta, von denen Porzig (1969: 263) meinte, sie seien die ,Vergegenständlichung eines Satzinhalts‘, von Substantivgruppen oder von in 
vergleichbarer Weise informationsverdichtenden drei- und mehrgliedrigen Komposita (,Enzym-Substrat-Komplex') ist eher ein Kennzeichen der kompakten, satzverkürzenden wissenschaftlichen Sprache;

an die Stelle eines ökonomischen Schematismus, z.B. der Neigung zu stereotypen Satzbauplänen, tritt in der populären Prosa eine Tendenz zur Variation, zur rhythmischen Abwechslung; der Satzbau ist aber relativ einfach und überschaubar; lange und informationskompakte Sätze werden, wie wir oben gesehen haben, aufgelöst.

Nach den Bemerkungen zur Syntax nur noch einige wenige zum Substantiv:

die Zahl der Nomina tritt zurück, der Stil ist eher verbal;

die Zahl der Symbole und wissenschaftlichen Termini, auch der Fremdwörter, ist ungleich geringer;

an die Stelle des abstrakten und sachlich bezeichnenden Substantivs tritt recht oft das anschauliche, suggestive, mit dem Gefühle und Wertungen assoziiert werden; dabei ist wiederum die Personifizierung und Anthropomorphisierung der auffälligste Zug.

Am Beispiel des Bildlexikons Die Naturwunder der Erde lässt sich gut sehen, wie ein solches Werk Übersetzungsarbeit leistet. Die einzelnen Einträge sind stark von nicht eigens erklärtem geologischem Fachvokabular durchsetzt. In dem Artikel ,Eiger‘ findet sich z. B. der Satz: „Während die starre Gneis- und Granitmasse der Zentralalpen ungefaltet blieb, wurden die weicheren Sedimentschichten des Perms, des Trias und des Juras im Verlauf der tertiären Gebirgsbildung gegen den kristallinen Block in verwickelte Falten geworfen." Die Termini sind in der ,Erklärung wichtiger Fachausdrücke“ am Schluss des Bandes klar und gemeinverständlich erläutert. Charakteristischer ist die indirekte Übersetzungsarbeit, die das Werk leistet. Schon die Fachsprache der Geologie ist oft bildhaft; dieser Zug ist in dem Bildlexikon sehr verstärkt. Bildhafte Adjektive, Verben und vor allem auch Substantive, in deren Erfindung das Werk besonders produktiv zu sein scheint, leisten eine plastische Vergegenwärtigung, die ermöglicht, dass unversehens eine Menge geologischen Wissens und Fachvokabulars begriffen und erlernt wird. („Danach folgt flüssige Lava, die jedoch hauptsächlich aus den fast 300 Nebenkratern an den Flanken des Bergkegels hervorquillt. Die meisten dieser parasitären Kleinvulkane sitzen auf nordsüdlich verlaufenden Spalten, die während der Ausbruchsphasen aufreißen.“)

Dem zuletzt Gesagten entspricht, wo abstrakte Zusammenhänge dargestellt werden, der Gebrauch des Beispiels. In dem oben erwähnten Buch Meilensteine des 20. Jahrhunderts gelingt es z.B. in dem Kapitel „Raum, Zeit und Einstein“ (44ff.) die theoretischen Konzepte Einsteins in einem nicht geringen Grad begreiflich zu machen, indem sie an Hand einer Reihe von Versuchen, Gedankenexperimenten und Vergleichen, meist den von Einstein selbst benutzten, zu einer 
möglichst weit vorgetriebenen Anschauung gebracht werden. In gleicher Weise gewährt das spätere Kapitel „Freud erschließt das Unbewußte“ (60ff.) einen anschaulich informativen Einblick in die Psychoanalyse. Die an sich schon sehr gleichnisreiche Sprache Freuds erscheint auf der Stufe noch handgreiflicherer Bildlichkeit: „Träume wirken wie Sicherheitsventile: Sie stellen den Versuch der Psyche dar, emotionale Spannungen zu lösen, ohne das wache Bewußtsein zu beanspruchen. Die einfachste Form solcher Entspannung ist die Wunscherfüllung. So träumt etwa der hungrige Schläfer, er nehme ein opulentes Mahl ein.“ (62)

Schon an kleinen sprachlichen Einzelbeobachtungen zeichnet sich ab, in welcher Weise sich beim Übergang von der wissenschaftlichen in die populäre Prosa etwas verschiebt: In Richtung auf höhere Redundanz, emotional getönte und anschauliche Sprache (das beides gehört wohl zusammen), dynamisierende Elemente und Vermenschlichung. An der Art der verwendeten Adjektive, Verben, Substantive ließe sich der Grad der angestrebten Popularität ablesen.

Betrachtet man die Texte von dem übergeordneten Gesichtspunkt der Gattung aus, so erscheint die wissenschaftliche Darstellung als relativ einheitlich, die populäre wird ergänzt und durchmischt von anderen Gattungen:

von der eigenen Gattung bildlicher Darstellung, sei das optische Zeichen nun mehr informativ oder Stimmungsträger;

vom verbalen Stimmungsbild - das Kapitel über die Russische Revolution in Meilensteine (134) beginnt mit der Schilderung wochenlangen Regens in Petrograd, bis ein Sonnenstrahl durch die Wolken bricht;

vor allem sind es erzählende Gattungen: der Autor knüpft an ein Erlebnis an, geht von einer Anekdote aus, die ein Schlaglicht auf sein Thema wirft, er vermittelt den Stoff, indem er das Leben eines Forschers und die Geschichte seiner Entdeckungen erzählt oder allgemein die Entwicklung des Wissens auf einem Gebiet. Der Autor populärwissenschaftlicher Werke erzählt überhaupt gern Geschichten; wir kommen darauf zurück.

Das letztere ist insbesondere ein Kennzeichen des sogenannten ,erzählerischen Sachbuchs‘. Zu diesem Typ gehört Hoimar von Ditfurths Im Anfang war der Wasserstoff (zuerst 1972, zitiert wird nach der Taschenbuchausgabe 1975). Das Buch erzählt die Geschichte der Welt von der Explosion des Weltalls, dem Urknall, über die Entstehung des Lebens, dem Auszug aus dem Wasser und die Entwicklung von Bewusstsein bis in die intergalaktale Zukunft. Von Ditfurth (1975: 72, 252) nennt sein Buch eine ,Nacherzählung‘.

Im Gegensatz zu anderer Sachliteratur ist in einem solchen Werk ein individueller Autor durchaus erkennbar; aber auf der anderen Seite verwendet gerade von Ditfurth die typischen Mittel der Übertragung zahlreich und auf vielseitige Weise. Ich versuche, von Ditfurths Buch näher zu charakterisieren, indem ich das 
Basisfunktionen-Modell der Sprache als ordnendes Instrument verwende. Die Besonderheit eines Textes ergibt sich nach Roman Jakobson daraus, dass die Grundfunktionen der Sprache in hierarchischer Ordnung auftreten und die dominierende Funktion die verbale Struktur bestimmt. ${ }^{7}$

Wir stellen also vor dem kontrastierenden Hintergrund des idealisierten wissenschaftlichen Darstellungstypus folgende Frage: In welcher Weise und mit welcher Auswahl sprachlicher Mittel werden die Basisfunktionen der Sprache hier wahrgenommen?

\section{Die Verwendung der Basisfunktionen der Sprache und zugehöriger sprachlicher Mittel in Hoimar von Ditfurths Im Anfang war der Wasserstoff}

Wir können, Bühler, Kainz und Jakobson folgend und erweiternd, acht Basisfunktionen der Sprache unterscheiden. Gehen wir sie im Einzelnen durch:

\section{Die Funktion der Darstellung und Unterscheidung}

Die wissenschaftliche Sprache, speziell die naturwissenschaftliche, ist per Definition sachliche Darstellung. Ihre Sachbezogenheit äußert sich im Vermeiden von Redundanzen, Klarheit und Exaktheit der Unterscheidungen, Ökonomie. Dem entsprechen ihre Mittel: vereinbarte und definierte Termini und Nomenklatur, charakterisierende und orientierende Adjektive und Substantive. Recht typisch sind die schon erwähnten sogenannten ,blassen' Verben, die fast nur noch eine grammatische, satzkonstituierende Funktion haben, und informationsverdichtende Mittel wie der Gebrauch des Passivs und des Partizips, der Abstrakta und Substantivgruppen, der mehrgliedrigen kompakten Komposita. - Gewisse ,Topoi kommen hinzu, denen oft idiomatische Ausdrucksweisen entsprechen: Formen, in denen man einen Begriff definiert, eine Aussage begründet oder belegt, ihren Gültigkeitsgrad einschränkt, aber auch die Wendungen, in denen wir den Stand

7 Jakobson 1964: 535. - Im folgenden Abschnitt führe ich weiter aus, was ich in „Probleme der Sprachdifferenzierung und Sprachvereinheitlichung. Entfernung der Fachsprachen von der Gemeinsprache und ihre ,Übersetzung“ durch populärwissenschaftliche Literatur“ (Pörksen 1983a) skizziert habe. 
der Forschung festhalten und unsere Äußerungen in den Fortschrittsstrom der Wissenschaft einbetten. Von Ditfurths Text ist überaus redundant. Der Autor wiederholt viel, verdeutlicht. ,Mit anderen Worten', heißt es z.B., ,anders ausgedrückt‘. Viele seiner Sätze bringen keine neue Information. Die Funktion der sachbezogenen unterscheidenden Darstellung und ihre typischen Mittel, Terminologie und satzverkürzende Formen, treten zurück oder werden aufgelöst in andere Funktionen. Gegenüber dem Nominalstil ist von Ditfurths Stil stärker verbal; an Stelle der ,blassen', funktionalisierten Verben bevorzugt er solche, die innerhalb des Satzes geschehen- und bildkonstituierende Wirkung haben; der personifizierende, anthropomorphisierende Bildgehalt ist typisch: „Die Natur hat schon vor vier Milliarden Jahren eine Technik entwickelt, um mit der Aufgabe fertig zu werden, ohne deren Lösung die Entstehung von Leben undenkbar geblieben wäre. Die Mittel, deren sie sich bedient [...]. “ Man würde in einem wissenschaftlichen Text eher Vorgangs- bzw. Zustandsverben erwarten oder doch die Passivkonstruktion. Von Ditfurth gebraucht das Tätigkeitsverb im Aktiv, die Sachzusammenhänge werden dynamisiert und nicht statisch oder vorgangshaft abgebildet.

\section{Funktion der Gliederung}

Die Gliederung hat als eine Technik der Darstellung eigenständiges Gewicht. Es scheint hier grundsätzlich zwei Möglichkeiten zu geben: einmal das deduktive Verfahren. Der Autor geht von Ergebnissen aus, stellt sie voran als etwas Fertiges, macht Voraussetzungen des Wissens, zu denen $u$. U. auch die eingeführten Termini und Nomenklaturen gehören, lässt die Begründungen zurücktreten und entwickelt seine Thesen, indem er meist vom Allgemeinen zum Speziellen vorangeht. Diesem deduktiven Verfahren steht das induktive gegenüber: der Autor macht wenig Voraussetzungen, $u$. U. kaum terminologische, knüpft an allgemein Bekanntes an und erweitert dessen Bezirk schrittweise um neue Tatsachen, die eine Erklärung verlangen, diskutiert sie, beteiligt also den Leser an der Erkenntnis- und Entdeckungsprozedur. Freud (1940ff., XVII: 141f.) beschreibt diese beiden Verfahren als ,dogmatische“ und ,genetische“ Methode. In der Wissenschaft überwiegt wohl der systematische deduktive Lehrvortrag, im erzählerischen Sachbuch das historisierende genetische Verfahren. Auch von Ditfurth bevorzugt diese Technik. Sie ist erzählerisch und sehr redundant, kommt dem Fassungsvermögen des nicht vorgebildeten Lesers entgegen und erhält, als Methode der schrittweisen verzögerten Enthüllung, die Spannung bis zum Schluss aufrecht.

Schon seinen Stoff, die Ergebnisse von Astrophysik und Geophysik, Chemie, Biologie, hat von Ditfurth in einen geschichtlichen Prozess eingeschmolzen, indem er ihn zu einem Roman des Alls aufarbeitet. 
Aber auch im Einzelnen geht er genetisch vor. Das erste Kapitel beginnt: „Im Frühjahr 1965 hörten Arno A. Penzias und Robert W. Wilson als erste Menschen das Echo der Entstehung der Welt - nur: sie wußten es nicht“ (von Ditfurth 1975: 15). Am Anfang steht das Rätsel und schrittweise erfährt der Leser, an dem Weg der Forschung entlanggeführt, seine Lösung.

Zwischen den Stoff und das Publikum tritt eine Figur, ihr Erkenntnisprozess als Mittler. Diese Figur kann bei von Ditfurth die personifizierte erkennende Menschheit sein, die Forschung, ein einzelner Forscher wie z. B. Giordano Bruno oder Einstein. Sie wird dem Publikum angenähert. Auch Einstein konnte sich keinen gekrümmten Raum vorstellen (von Ditfurth 1975: 31) - das ist beruhigend für uns alle. Schließlich ist der Autor selbst diese Identifikations- und Induktionsfigur. Er tritt immer wieder hervor, durch Ankündigungen oder Zusammenfassungen, als sichtbarer Arrangeur und als Erarbeiter seines Stoffes.

Dem hier beschriebenen Verfahren begegnen wir immer wieder: in Dieter Kuhns Wolkenstein ist der fachfremde recherchierende Autor die Brücke zwischen Publikum und Oswald, in dem Werk von Watson über die Doppel-Helix sind es zwei sehr menschlich geschilderte Forscher usw.

Bei von Ditfurth lässt sich diese Methode der schrittweisen verzögerten Enthüllung bis in die syntaktische Struktur beobachten. Er nutzt den Charakter des deutschen Satzes als Spannungsfeld, in dem der höchste Mitteilungswert, das Informationsziel, am Ende steht, geschickt aus. Die am Ende - oft nach Einschüben - gegebene Information ist u. U. nur ein Appell („,Der nächste Schritt, der sich wiederum aus [...] ergibt, ist in seinen Konsequenzen atemberaubend“; von Ditfurth 1975: 73), oder sie ist gering (,Enzyme sind Eiweißkörper ganz bestimmter Bauart.“) Der Leser erfährt, was er schon weiß, und ein kleines bisschen mehr. Eine appellierend angekündigte rätselhafte Mitteilung erhält häppchenweise eine gewisse Deutlichkeit und erst am Schluss einen prägnanten begrifflichen Umriss.

\section{Funktion der Metasprache - der Verständigung über den Sprachgebrauch}

Zur wissenschaftlichen Darstellung, insbesondere der systematisch-lehrhaften, gehört die als bekannt vorausgesetzte Terminologie und die explizite Vereinbarung von Nomenklatur und Begriffsinhalten. Auch von Ditfurths erzählerisches Sachbuch verwendet einen Teil seiner Arbeit darauf, einige wissenschaftliche Termini einführend zu vermitteln, aber die größte Energie richtet sie umgekehrt auf die Übertragung von wissenschaftlichen Termini und ihren Inhalten in die Gemeinsprache. Hier liegt das Zentrum der - im genauen Sinn des Wortes Übersetzungsarbeit. 
Welche Mittel werden bei der Einführung und Übersetzung verwandt?

z. B. die wörtliche Übersetzung - „was die Wissenschaftler als Photodissoziation (wörtlich ,Zerlegung durch Licht‘) des Wassers bezeichnen“ (von Ditfurth 1975: 71);

die Ersetzung des aus fremdsprachlichem Material gebildeten Terminus durch den erklärenden, durchsichtigen, definierenden Ausdruck - ,Biopolymere‘ durch ,Lebensbausteine‘;

die gemeinverständliche Definition - der Begriff ,Katalysatoren“ wird eingeführt: „Darunter versteht der Chemiker Substanzen, die in der Lage sind, chemische Reaktionen in Gang zu setzen oder zu beschleunigen, ohne daß sie selbst in die neu entstehende Verbindung eingehen [...]“;

die Synonymenvariation - der Begriff ,Erosion` z.B. wird durch bedeutungsähnliche Wörter eingekreist. „Wind und Regen hatten eben erst mit der Zerkleinerungsarbeit, der Erosion, begonnen, mit der Verwitterung, welche die Oberfläche des Urgesteins langsam in Staub und Sand verwandelte“ (von Ditfurth 1975: 69, Herv. von mir; U.P.);

die Paraphrase - von Ditfurth (1975: 65) spricht von der „langen Epoche einer ,abiotischen Genese organischer Makromoleküle““ und setzt hinzu: „auf deutsch also eine Zeit, in der alle die vielen komplizierten und empfindlichen organischen Moleküle entstanden waren, die gewissermaßen als Bausteine die Voraussetzung bildeten zur Entstehung der ersten, noch so primitiven lebenden Struktur“;

die Übersetzung des eigentlichen, abstrakten, neutralen Terminus in eine (meist anthropomorphe) Metapher - statt ,interplanetare Kontamination mit bakteriellen Keimen“ heißt es z.B. kurz „interplanetare Keimverschleppung“ (von Ditfurth 1975: 91) oder es ist von ,einer Art Totstellreflex` und einem ,Scheintod‘ der Bakterien im Weltraum die Rede (von Ditfurth 1975: 117). Mit diesem Übersetzungsmittel berührt sich eng die Ersetzung des sachorientierenden durch den konnotathaltigen, ausdrucks- und appellstarken, je nach Popularitätsstufe umgangssprachlichen oder auch jargonhaften Ausdruck - den wissenschaftlich üblichen Ausdruck ,Expansion des Weltalls‘ ersetzt von Ditfurth meist durch ,Explosion des Weltalls', und an Stelle des Terminus ,Urexplosion“ des Weltalls bevorzugt er die Ausdrücke ,Urknall‘ und ,Big Bang‘. Von Ditfurths Buch ist ein vielseitig angelegter Versuch, ein naturwissenschaftliches Weltbild der Gegenwart gemeinsprachlich bzw. bildungssprachlich auszuarbeiten - ein Versuch, dem man in unserer Belletristik m.W. kaum noch begegnet. 


\section{Die Funktion der fragenden und hypothetischen Erweiterung des gegebenen Horizonts}

Ein konstitutives Element wissenschaftlicher Arbeit ist wohl die Fragehaltung. Erweiterung des Wissens über Gegenstände und Sachzusammenhänge, Erforschen des Unbekannten. Die Sprache der Erweiterung des gegebenen Horizonts die Frage, der Konjunktiv und überhaupt das Modalfeld der Sprache, durch das wir den Sicherheitsgrad einer Aussage einschränken oder sie als nur denkmöglich hinstellen - ist daher ein wiederkehrendes Merkmal wissenschaftlicher Texte.

Diese Sprache ist in der für die Allgemeinheit geschriebenen Sachliteratur anscheinend viel seltener, sie will vor allem das gesicherte Wissen vermitteln und hat die Tendenz, Wissen als gesichert zu vermitteln. In der populären Sachliteratur gibt es eine Tendenz, den Leser auf einen geschlossenen Horizont zuzubewegen.

Trotzdem findet sich hier und auch bei von Ditfurth diese Sprachfunktion, allerdings meist in der Weise, dass sie sich von der Orientierung an der Sache auf die am Leser verschiebt. Der Verfasser begibt sich auf den Wissensstand des Lesers und sucht dessen Horizont zu erweitern - seine Frage- und Suchhaltung ist, wie oft in der genetischen Darstellungstechnik, eine scheinbare. Sie ist ein Mittel der Auflockerung des Stoffs. (,Was der Erde jetzt noch fehlte, war eine Atmosphäre. Woher sollte sie kommen? Die Antwort ist ebenso einfach wie verblüffend: Die Erde hat sie ausgeschwitzt“; von Ditfurth 1975: 59).

\section{Die Kontaktfunktion}

Dass die Kontaktfunktion direkt wahrgenommen wird, ist für wissenschaftliche Darstellung kaum typisch. Das Personalpronomen ,wir - als Autorenplural oder als Bezeichnung der Gemeinschaft von Autor und Leser - ist eine der wenigen direkten Formen.

Von Ditfurth benutzt das, wir‘ auf eigentümlich gestufte Weise:

er gebraucht, selten, den Autorenplural (die „Geschichte, die wir in diesem Buche zu rekonstruieren versuchen“; von Ditfurth 1975: 54);

gelegentlich ein ,wir‘, das für die Wissenschaft steht („Heute wissen wir allerdings, daß auch die Jeanssche Begegnungs-Theorie nicht stimmt“; von Ditfurth 1975: 50);

etwas öfter das ,wir‘, das Autor und Leser zusammenschließt („,Sehen wir uns näher an, was damit gemeint ist,“; von Ditfurth 1975: 48, ,jetzt wollen wir einmal annehmen [...]“; von Ditfurth 1975: 29);

am auffälligsten ist aber das umspannende, wir‘, durch das der Autor die Menschheit als Wissens- und Suchgemeinschaft vereinigt (,Wir haben uns im 
Verlaufe der Erforschung der Natur in den letzten Jahrhunderten daran gewöhnen müssen, daß die Wirklichkeit anders ist, als wir geglaubt hatten“, von Ditfurth 1975: 28). Dem entspricht der weitgespannte Gebrauch des besitzanzeigenden Fürworts (,unsere Erde‘, ,unser Sonnensystem“; von Ditfurth 1975: 52, ,unser Planetensystem'; von Ditfurth 1975: 45, ,unsere kosmische Heimat‘; von Ditfurth 1975: 54).

Die Kontaktfunktion wird vor allem indirekt, auf dem Umweg über andere Basisfunktionen wahrgenommen. Das gilt in von Ditfurths Fall nicht nur für die Art, wie er die bisher besprochenen typisch wissenschaftlichen Funktionen der Sprache didaktisch handhabt, sondern auch dafür, wie er auf dem Weg über sprachliches Ausdrucks- und Appellverhalten und durch poetische, ästhetische Mittel seinen Stoff den Bedürfnissen und Wünschen des Publikums annähert.

\section{Die Ausdrucksfunktion}

Der Autor dieses erzählerischen Sachbuchs sagt ,ich“ („Ich ertappe mich allerdings, wie ich gestehen muß, gelegentlich bei dem Gedanken [...]“; von Ditfurth 1975: 32), er drückt seine Subjektivität aus; von seinem Ich ausgehend, spricht er Gefühle und Wertungen aus, durch Stellung nehmende Modalwörter wie ,leider (von Ditfurth 1975: 48), ,endlich“ (von Ditfurth 1975: 52), oder durch von einer anthropozentrischen Position aus urteilende, wertende Adjektive: das Bild von der Erde in der Wasserdampfzeit ist ,alptraumhaft‘ (von Ditfurth 1975: 62), das Milieu der Venus ,höllisch“ (von Ditfurth 1975: 55), und unser Planet ist ,angenehme‘ 150 Millionen Kilometer von der Sonne entfernt (von Ditfurth 1975: 58). Es gibt Ausrufe (von Ditfurth 1975: 48), etwas ist ,atemberaubend“ (von Ditfurth 1975: 59), es verursacht Herzklopfen (von Ditfurth 1975: 75).

So wie er es schon als der zwischen Stoff und Publikum vermittelnde Erzähler ist, wird der Autor noch mehr durch sein Ausdrucksverhalten zur Identifikationsfigur für den Leser.

\section{Appellfunktion}

Ausdruck und vor allem Appell, der den Leser zu reizen und in seinem Verhalten zu steuern sucht, sind traditionellerweise am wenigsten typisch für einen wissenschaftlichen Text: in der populären Prosa treten sie um so deutlicher hervor.

Der Appellcharakter der Sachliteratur - schon das Wort ,Sache“ erhält in diesem Kontext einen Appellwert - lässt sich am krassesten an den Titeln ablesen. Bestimmte Wortfelder kehren wieder: 
sie sprechen das Bedürfnis nach räumlicher, globaler Weite an (Kinder des Weltalls, Welt und Wasser, Zwischen den Kontinenten) und nach geschichtlicher Ferne, den Sinn für das Ferne, Fremde, Exotische;

sie spekulieren auf das Interesse am ,Rätsel', ,Geheimnis‘, ,Wunder‘ (Wunder und Rätsel der Pyramiden, Wundermaschine Mensch, Das Geheimnis der Maja);

sie appellieren an den Stolz auf die menschliche Erkenntnisleistung und an einen ungebrochenen optimistischen Fortschrittsglauben, und auf der anderen Seite an den Pessimismus, das apokalyptische Weltgefühl (aus Oswald Spenglers Die Morphologie der Geschichte machte ein pfiffiger Verleger Der Untergang des Abendlandes).

Auch von Ditfurths Buch ist eine vielschichtige Appellstruktur unterlegt:

Der Titel Im Anfang war der Wasserstoff ist eine Prestigeanleihe und auch als Kontrafaktur zu dem bekannten Bibelwort eine provozierende Antithese.

Von Ditfurth lehnt sich auch sonst an gängige idiomatische Redewendungen und bekannte Titel an, so in seinen Kapitelüberschriften: ,Ein Platz an der Sonne،, ,Fiel das Leben vom Himmel?‘, ,Auszug aus dem Wasser‘, ,Älter als alle Gehirne‘. Es ist das Schlepptauprinzip der Reklame und wie dort oder auch sonst in der Titulatur sind das Paradox (,Geschichte der Zukunft` - ,Zurück zu den Sternen`) und der weitgespannte Kontrast (,Programme aus der Steinzeit‘ - ,Wiege im Weltall`) beliebte Mittel.

Die ,Topoi‘ der wissenschaftlichen Darstellung gewinnen in einem solchen Zusammenhang eine teilweise Appellfunktion. Wendungen wie z.B. ,wie wir heute wissen“ (von Ditfurth 1975: 19), ,nach allem, was wir wissen“ (von Ditfurth 1975: 39), ,wie auch die Wissenschaft noch vor kurzem glaubte، (von Ditfurth 1975: 64), die überraschende Einführung eines Terminus vermitteln das Ansehen von Wissenschaftlichkeit.

An die Stelle wissenschaftlichen Begründens und Belegens setzt sich als allgemeine Form, zu beglaubigen und zu legitimieren, das Autoritätenzitat (,die Wissenschaft', ,die Forschung‘, ,der Chemiker‘, ,Nobelpreisträger Lorenz‘, ,die berühmte Princeton-Universität‘). ${ }^{8}$

Die Tendenz vom informierenden, charakterisierenden und orientierenden zum stellungnehmenden, urteilenden und wertenden, ausdrucks- und appellhaltigen Wort ließe sich an ungezählten Adjektiven belegen, an nur unterstreichenden Superlativen, an dem oft bildhaften und mehr suggestiven Charakter der

8 Auf einem niedrigeren Niveau des populären Sachbuchs kommt es häufig vor, dass die wissenschaftlichen Darstellungsmittel fast nur noch Appellwert haben. Der Lesetext ist eingängig, Zahlen werden hier abgerundet, daneben gibt es, eingerahmt in Boxen, Diagramme, Tabellen und mehrstellige Zahlen hinterm Komma; sie sichern das Prestige der Wissenschaftlichkeit. 
Substantive. Von den anschaulichen, sehr oft anthropomorphisierenden Verben der Aktion war schon die Rede.

Eine Appellfunktion nimmt von Ditfurth aber auch von einem höheren Standpunkt aus wahr. Er möchte keineswegs nur naturwissenschaftliche Erkenntnisse vermitteln, sondern sein aus der Naturwissenschaft gewonnenes Weltbild. Der seit dem 18. Jahrhundert sich vollziehende Rollenwandel des wissenschaftlichen, insbesondere naturwissenschaftlichen Autors, der - an Stelle von Religion und Philosophie - die Aufgabe der allgemeinen Weltinterpretation übernimmt, ist an dem Buch deutlich zu beobachten.

\section{Poetische Funktion}

Der naturwissenschaftliche Text gilt als ästhetisch neutral; die populäre Sachprosa ästhetisiert, poetisiert oft.

Wir können eine Ästhetisierung des von Ditfurthschen Textes an vielen Kleinigkeiten beobachten:

auf der lautlichen Ebene - in der Alliteration z.B. (er spricht von der Atmosphäre, welche der „erkaltenden Kruste entquoll“; von Ditfurth 1975: 76);

auf der syntaktischen Ebene - durch den Anschluss an gängige Rhythmen („Älter als alle Gehirne“ heißt eine Kapitelüberschrift, „Geschichte der Zukunft“), durch die Variation der Satzbaupläne. Gegenüber der Ökonomie und dem häufigen Schematismus wissenschaftlicher Syntax und Wortwahl ist die Variation ein durchgängiges Stilprinzip;

auf der semantischen Ebene - die leichten semantischen Regelverletzungen (,Kleine, grüne Sklaven“, ,Nachricht vom Saurier' sind andere Kapitelüberschriften). Die Metaphorik könnte ein eigenes Kapitel beanspruchen; auch am Bildmaterial dieses Buches ließe sich einiges zeigen: die Bilder, meist Kombinationen von Zeichnung und Farbe, sind recht informativ, teils aber auch ästhetisch einstimmend oder, wie das rote Wasserstoffatom auf dem Umschlag, aggressiv appellierend.

Das Buch ist wirkungsbewusst und auf Schönheit hin konzipiert; die Funktionen der Formung und des Appells sind oft verbunden, wenn ein Autor sich rhetorischer Figuren bedient. Rhetorisierung ist ein Kennzeichen dieses Werks und überhaupt der sogenannten ,Sachliteratur‘. Die Sache wird amplifiziert und vereindringlicht. Von Ditfurth bedient sich des Instrumentariums der Rhetorik auf breiter Ebene: des Ausrufs, der Frage und des Kurzdialogs (von Ditfurth 1975: 59), der Sentenz (von Ditfurth 1975: 63), der Metaphern und Personifizierungen und Vergleiche (von Ditfurth 1975: 31). Er beginnt mit einer ,similitudo‘, dem Vergleich zwischen einer Filmszene - der raffinierten Flucht eines Verbrechers in ein 
Spiegelkabinett - mit der rätselhaften Fähigkeit der Kaiserraupe, sich durch Attrappen zu schützen (von Ditfurth 1975: 7ff.), und er beherrscht das klassische Argument der ,refutatio‘: das rationale Pathos des Autors gewinnt Profil, indem er sich gegen die Nichtigkeit der aufklärungsfeindlichen gegnerischen Mehrheit wendet (von Ditfurth 1975: 77ff.).

Wir fassen zusammen:

Die wissenschaftliche Sprache ist gekennzeichnet durch eine strenge Auswahl aus den Basisfunktionen der Sprache, und die Mittel, in denen sie wahrgenommen werden, sind relativ begrenzt und z. T. genormt oder standardisiert. Die bevorzugten Funktionen sind die ersten vier - Darstellung und Unterscheidung, Gliederung, Horizonterweiterung, Metasprache - und sie werden vorwiegend sachorientiert gehandhabt.

Der populärwissenschaftliche Text zeichnet sich aus durch Breite. In ihm werden alle Basisfunktionen der Sprache wahrgenommen und die typisch wissenschaftlichen auf veränderte Weise. Das Spektrum der eingesetzten sprachlichen Mittel ist weit, und diese selbst sind variabel.

Die Verschiebung von der Sachorientierung auf die Publikumsorientierung, von der anfangs die Rede war, wird an der Verschiebung beim Gebrauch der Basisfunktionen erkennbar: die vier vorwiegend kommunikativen, empfängerorientierten Funktionen - Kontakt, Ausdruck und Appell, poetische Form - gewinnen an Bedeutung und die typisch wissenschaftlichen werden kommunikativ gehandhabt.

Die Mittel, mit denen ein wissenschaftlicher Text in einen populären übersetzt bzw. umgesetzt wird, konvergieren in Redundanz und Dynamisierung, Veranschaulichung und Emotionalisierung, sie vermitteln dem Leser die Möglichkeit, sich zu identifizieren, und zielen auf Vermenschlichung.

Eine Wissenschaftssprache ist denkbar - und es gibt dafür auch Beispiele -, in der die Grundfunktionen der Sprache vollständig wahrgenommen werden, ohne dass eine durchgreifende Sachbezogenheit verlorengeht.

Die erwähnten Funktionen und zugehörigen sprachlichen Mittel können auf unterschiedlicher Stufe, je nach Grad und Umfang der angestrebten Popularität, gehandhabt werden. In von Ditfurths Buch wird eine Menge wissenschaftlicher Erkenntnisse für ein bildungssprachliches Publikum übersetzt und übertragen Züge der Einbettung und Auflösung in die empfängerorientierten Funktionen sind andererseits deutlich.

Wo ein sehr viel breiteres Publikum und ein massenhafter Absatz das Ziel ist, wird sich der Gebrauch der Basisfunktionen und sprachlichen Mittel weiter in der angegebenen Richtung verschieben: die Sache und ihre gedankliche Erarbeitung treten weiter zurück hinter die Reize des Ausdrucks, des Appells und der Poesie. 
In seinen Schriften zur Sprache forderte Leibniz, sich nicht - wie die Sprachgesellschaften des Barock - auf ,Klinggedichte،, sondern auf die sprachliche Ausarbeitung der Sachgebiete zu konzentrieren, um dem Zustand der Sprache seiner Zeit aufzuhelfen (Leibniz 1916a: 15, 24). Er entdeckte die Sachprosa als Mittel der Spracherziehung und forderte einen Kanon vorbildlicher ,Kernschriften'.

Tatsächlich ist der Kanon, der für unsere Einheitssprache normbildend wirkte, dann ganz überwiegend ein literarischer gewesen: er bestand aus den Werken unserer Klassik. Deren Sprache weist, von der Gegenwart her gesehen, bei aller Vorbildhaftigkeit große Lücken auf - es fehlen weite Sachbereiche, wie z.B. die der Wirtschaft, der Technik, der Naturwissenschaften - und diese Lücken sind wohl auch kaum zu schließen durch die Belletristik der Gegenwart. Wäre es nicht richtig, den Gedanken der sprachbildenden Wirkung der Sachprosa mehr als bisher zu beherzigen und einmal ein Lesebuch hervorragender wissenschaftlicher, insbesondere naturwissenschaftlicher Prosa - von Linné bis zu den Autoren des 20. Jahrhunderts - zusammenzustellen? 